\title{
Débats monétaires autour de la dévaluation du
} franc de 1969

\section{Bertrand Blancheton et Christian Bordes}

\section{(2) OpenEdition}

\section{Journals}

Édition électronique

URL : http://journals.openedition.org/ress/236

DOI : $10.4000 /$ ress.236

ISSN : 1663-4446

Éditeur

Librairie Droz

Édition imprimée

Date de publication : 1 juillet 2007

Pagination : 213-232

ISBN : 978-2-600-01155-6

ISSN : 0048-8046

Référence électronique

Bertrand Blancheton et Christian Bordes, « Débats monétaires autour de la dévaluation du franc de 1969 », Revue européenne des sciences sociales [En ligne], XLV-137 | 2007, mis en ligne le 01 juillet 2010, consulté le 19 avril 2019. URL : http://journals.openedition.org/ress/236 ; DOI : 10.4000/ ress.236 


\section{Bertrand BLANCHETON et Christian BORDES}

\section{DÉBATS MONÉTAIRES AUTOUR DE LA DÉVALUATION DU FRANC DE 1969}

L'originalité de cette contribution sur la dévaluation du franc de 1969 est de chercher à évaluer l'étroitesse des liens - par nature multiples et complexes entre théories et pratiques monétaires, de se centrer ouvertement sur les interactions entre analyses et faits économiques. Pour ce faire, deux objectifs doivent être poursuivis en parallèle.

L'étude entend tout d'abord analyser l'évolution du débat théorique sur la question de l'ajustement de la balance des paiements. Dans cette perspective, nous souhaitons estimer l'influence éventuelle des événements monétaires français de la fin des années 1960 sur les développements de l'analyse économique. Au sein du régime de Bretton Woods les cas de dévaluation de « grandes monnaies » restent rares ; le double décrochage de la livre sterling en 1967 et du franc français en 1969 est susceptible d'avoir dynamisé le débat sur l'ajustement. A défaut de voir les événements français comme un tournant majeur vers des recompositions analytiques, nous pourrons - à tout le moins - nous en servir comme d'un prisme pour étudier la question.

Ce travail cherche ensuite à mettre en exergue les fondements théoriques des conceptions des principaux acteurs de la politique monétaire française (gouvernement, direction du Trésor, Banque de France et autres conseillers), c'est-à-dire l'impact des avancées analytiques sur la conduite des affaires. Si, à première vue, il peut sembler que le refus de la dévaluation en novembre 1968 et la décision de dévaluer d'août 1969 relèvent d'une logique « purement politique » ${ }^{1}$, il convient de ne jamais perdre de vue les propos de Keynes, qui en conclusion du chapitre 24 de la Théorie Générale affirmait : "Les idées, justes ou fausses, des philosophes de l'économie et de la politique ont plus d'importance qu'on ne le pense en général. A vrai dire le monde est presque mené par elles. Les hommes d'action qui se croient parfaitement affranchis des influences doctrinales sont d'ordinaire les esclaves de quelque économiste passé. Les visionnaires influents, qui entendent des voix dans le ciel, distillent des utopies nées quelques années plus tôt dans le cerveau de quelque écrivaillon de Faculté » (1936, 1971, p. 376). Nous chercherons à mettre en exergue ces influences diffuses et lointaines en revenant sur les prises de position des principaux acteurs de cet épisode de notre histoire monétaire. Nous n'oublierons pas que les références du personnel politique à une théo-

1 Comme en 1936, en 1958 ou plus tard en 1981 (Asselain, 2002), la dévaluation constitue $a$ priori en un moyen d'apurer le passé et de faire porter la responsabilité d'une «perte de substance » monétaire sur le précédent régime. 
rie économique peuvent être de nature circonstancielle et ne sont toujours que partielles, ancrées à une idée simple, une intuition de bons sens. Par extension, l'un des enjeux sera pour nous de mettre au jour les facteurs éventuels tant des divergences que des convergences de vues entre les pôles de l'autorité monétaire. De même, il conviendra de voir si l'on peut discerner certaines continuités historiques dans l'adhésion de tel ou tel acteur à une théorie particulière, notamment vis-à-vis du précédent de la dévaluation de 1958.

Notre démarche se déroule en trois temps. Nous mettons d'abord le sujet en perspective historique tant du point de vue de l'analyse de la question de l'ajustement que de celui des faits économiques français. Nous concentrons ensuite notre attention sur les événements des années 1968 et 1969 afin de déterminer l'influence des arguments économiques et des facteurs politiques sur les décisions monétaires. Enfin nous étudions la rétroaction des faits sur la théorie, principalement les progrès réalisés dans la connaissance des différentes phases du processus d'ajustement ainsi que les amendements apportés au modèle monétaire de la balance des paiements.

\section{MISE EN PERSPECTIVE HISTORIQUE}

Nous allons d'abord dresser un panorama des théories de l'ajustement de la balance des paiements au milieu des années soixante avant d'étudier l'influence de chacun de ces courants auprès des différents acteurs de la politique monétaire française.

\section{Panorama des théories de l'ajustement de la balance des paiements}

Dans les années 1960, l'on retrouve bien - dans ses grandes lignes - la traditionnelle opposition ${ }^{2}$ entre keynésiens et monétaristes à propos de l'origine d'un déficit de la balance des paiements : pour les premiers, les causes du déséquilibre sont à rechercher du coté de déterminants réels (le haut de la balance), pour les seconds, elles résultent de facteurs strictement monétaires (le bas de la balance). Mais la période étudiée présente une complexité particulière - due notamment à un regain d'intérêt pour cette question - qui appelle immédiatement un essai de clarification.

Au risque d'apparaître quelque peu caricatural, il nous semble que côté keynésien deux courants peuvent être distingués.

Fidèles à une lecture a priori littérale de Keynes ${ }^{3}$, les post-keynésiens de l'Ecole de Cambridge (parmi eux citons Kaldor et Robinson) sont partisans d'une

2 Aux dires de Kindleberger (1990, p.12), cette opposition trouve son origine au XVII ${ }^{\mathrm{e}}$ siècle, voire avant, et traverse depuis l'histoire monétaire et financière de l'Europe.

3 L'analyse de Keynes transparaît par exemple à travers le citation suivante extraite d'un article sur le problème des transferts allemands : «A un moment donné, la structure économique d'un pays, par rapport à la structure économique de ses voisins, permet d'atteindre un certain niveau "naturel" d'exportations. Opérer un changement de ce niveau par des actions délibérées est extrêmement difficile $(1929,1983$, pp. 457-458) 
approche structurelle de la balance des paiements. Dans cette perspective, le solde courant dépend des structures internes de la croissance (spécialisations avantageuses et porteuses, différenciations des produits, etc.). S'ils se situent dans une logique d'ajustement par les quantités, les Cambridgiens ne rejettent pas pour autant toute influence des prix : le nom de Joan Robinson est ainsi associé au théorème des élasticités critiques. Pour ce courant keynésien, lutter contre un déficit passe avant tout par la mise en œuvre d'une politique sectorielle sélective en matière d'investissements publics et la recherche de spécialisations dans des productions dont les demandes interne et externe sont peu élastiques au prix.

Dans la lignée des travaux de Hicks et Samuelson (sans doute convient-il aussi de citer Meade), les néo-keynésiens semblent faire une place plus directe aux prix des biens et services échangeables. L'on trouve ici l'intuition élémentaire qu'une dévaluation ou une dépréciation du change améliore - à court terme - la compétitivité des entreprises nationales et constitue un facteur de soutien de la demande, notamment par le jeu du multiplicateur. L'optique de l'absorption (Alexander) nous semble se situer dans cette veine. De même, le cadre IS/LM/FE sans fixité du change nous paraît constituer une formalisation de ce courant d'analyse. L'ajustement se fait donc ici encore par les quantités même si effet volume et effet prix se combinent au sein d'un processus qui n'a pas encore été étudié dans les détails à l'époque. Notons enfin que la baisse de la valeur externe de la monnaie nationale est sans conséquence inflationniste tant que des capacités productives restent inemployées.

Côté monétariste, à l'approche « métalliste » à la Rueff version moderne du «Price Specie Flow Mechanism» de Hume et Ricardo, s'est ajoutée, à partir de la fin des années 1950, l'approche dite monétaire de la balance des paiements.

Chez J. Rueff, dans le cadre d'un système d'étalon-marchandise et de changes fixes, le rééquilibrage de la balance revêt un caractère automatique. Un déficit s'accompagne d'un transfert de réserves métalliques vers l'économie étrangère qui a pour conséquences la hausse de la masse monétaire et, en vertu de la théorie quantitative de la monnaie, l'accroissement du niveau général des prix en son sein. La compétitivité prix des produits de cette même économie est réduite, ce qui conduit au rétablissement de l'équilibre externe.

$\mathrm{Au}$ moment où M. Friedman pose les bases de son analyse monétaire, une autre approche monétariste de la balance des paiements apparaît sous l'impulsion du département des études du FMI, alors dirigé par J. Polak. Elle sera ensuite développée par d'autres auteurs notamment Johnson, Frenkel ou Swoboda. L'on doit se situer ici au sein d'un régime de changes flexibles (la flexibilité est préférable à la fixité) où prévaut la relation de parité des pouvoirs d'achat. L'idée de base est que le solde courant d'un pays résulte d'un déséquilibre entre offre et demande de monnaie. Le déséquilibre externe est transitoire. Il entrâne en effet une variation du change puis du niveau général des prix, ce qui conduit in fine les agents à ajuster leur demande d'encaisse : sans viscosités de prix, le rééquilibrage de la balance est très rapide et la question de l'ajustement perd même sa nature politique.

De manière plus générale, là encore au risque de tomber dans la caricature, il nous semble que le débat sur l'ajustement de la balance des paiements peut 
renvoyer au statut des relations économiques internationales en matière de politique macro-économique, clivage fondamental entre veines dirigiste et libérale en histoire de la pensée et de l'analyse économique. Chez les économistes « dirigistes » (mercantilistes, puis keynésiens), les relations extérieures semblent au service de la poursuite des objectifs internes de politique économique. Par ses effets multiplicateurs, un excédent courant pourra soutenir durablement la demande, la production et l'emploi. Au contraire, pour les partisans du «laisser faire », il faut accepter toutes les implications de la contrainte extérieure, les ajustements internes qu'elle implique et notamment en histoire contemporaine (en changes fixes), le caractère endogène qu'elle confère à la politique monétaire. Cette approche suppose une action de sauvegarde de l'équilibre externe et l'acceptation du principe d'une croissance nationale fortement tributaire de la conjoncture mondiale. L'hypothèse d'une convergence des économies du fait d'un transfert de technologie et de la mobilité des facteurs de production justifie à long terme le caractère optimal de cette solution libre-échangiste.

\section{Ruptures et continuités monétaires d'une dévaluation à l'autre (1958-1969)}

La date de 1958 paraît s'imposer comme le point de départ d'une mise en perspective historique des événements monétaires français de la fin des années 1960. Le précédent d'une dévaluation réussie, la survenance d'un bouleversement institutionnel majeur, la genèse d'un régime monétaire encore en vigueur dans les années 1960, le renforcement d'une tendance à l'ouverture de l'économie française (contrainte fondamentale en matière de politique monétaire) ainsi que la longévité de nombreux acteurs (décideurs ou conseillers) de la politique économique invitent à ce choix.

La rupture institutionnelle qui survient en 1958 ouvre la voie à une plus grande cohérence de l'interventionnisme du pouvoir exécutif dans les affaires monétaires : le gouvernement dispose désormais du temps nécessaire à l'application efficace d'une politique économique et le Président de la République (d'abord - il est vrai - parce qu'il s'agit du général De Gaulle) devient l'arbitre suprême des débats monétaires.

En matière économique, De Gaulle ressent immédiatement la nécessité de mutations elles aussi profondes, mais il n'en trace pas lui-même les contours précis. Malgré les surprenantes hésitations de son ministre des Finances Antoine Pinay, il charge Jacques Rueff d'une mission globale de réflexion. Pourquoi ne pas avoir confié cette responsabilité aux personnels de la rue de Rivoli ? Pour de Gaulle les décisions à prendre sont alors "si étendues et si profondes qu'elles dépassent l'horizon du service normal » (cité par Lacouture, 1985, tome II, p. 668). La création du Comité Rueff peut être interprétée comme un pied de nez à une haute administration des Finances très dirigiste, pour qui la lutte contre l'inflation n'a jamais été une véritable priorité sous le précédent régime. On dit les hauts fonctionnaires keynésiens du fait de leur accoutumance à financer par l'emprunt un déficit budgétaire ouvertement accepté (voir Bévant, 1993, p. 472). Il apparaît ici que la préservation de la valeur externe de la monnaie ne serait pas 
historiquement l'objectif prioritaire de la rue de Rivoli et que, par conséquent, elle ne manifesterait pas une hostilité de principe à la dévaluation du franc.

Le Comité Rueff, réuni à trente reprises entre septembre et décembre 1958, propose des mesures de nature à rompre durablement avec l'instabilité financière et monétaire de la $I^{\mathrm{e}}$ République en même temps qu'il prépare une plus large ouverture de l'économie française à la concurrence internationale. Les dernières années de la IV ${ }^{\mathrm{e}}$ République ont en effet été marquées par la persistance d'un déficit budgétaire, sous l'influence notamment des «événements » d'Algérie. Son financement monétaire a largement contribué à l'apparition d'un différentiel d'inflation nuisible à la compétitivité des entreprises françaises. Une hausse du niveau général des prix de $16,2 \%$ et un taux de couverture des exportations de marchandises de $91 \%$ témoignent de l'intensité des déséquilibres interne et externe en 1958 d'autant plus que le rythme de croissance du PNB connaît cette année-là un ralentissement sensible ( $2,6 \%$, contre $6 \%$ en 1957), ce qui freine naturellement la hausse des prix et les importations.

Sous l'influence prédominante de Jacques Rueff, le Comité propose d'endiguer l'inflation en contractant la demande (réduction du déficit budgétaire) et en soulageant l'offre (désindexation salariale). Cette stabilisation doit permettre de faire face à une contrainte extérieure rendue plus forte du fait de l'application souhaitable du Traité de Rome qui prévoit en particulier une réduction substantielle des barrières douanières. Grâce notamment à la force de persuasion de Jacques Rueff bien sûr, mais aussi de Roger Goetze et de Georges Pompidou ${ }^{4}$, de Gaulle décide d'appliquer le plan du Comité - chose rare - dans son intégralité et dans un délai très court.

Encore fallait-il apurer complètement le passé. Le Comité Rueff propose également de dévaluer - de nouveau ${ }^{5}$ - le franc pour retrouver rapidement une marge de compétitivité : la dévaluation intervient le 27 du mois, le taux choisi est celui proposé par la Banque de France ${ }^{6}$, soit $15 \%$. Il est suffisamment élevé pour que l'économie nationale conserve une marge de compétitivité à la suite de la mise en œuvre d'une politique de vérité des prix qui prévoit notamment la suppression des subventions à l'exportation. De même, le premier janvier 1959 un nouveau franc voit le jour (1 nouveau franc $=100$ anciens francs). Ce changement revêt une portée symbolique certaine : vis-à-vis de l'opinion il sera désormais difficile de faire admettre l'abaissement de la valeur du franc. Pour le général de Gaulle, la grandeur de la France se confondra avec le maintien de l'intégralité de la valeur du « franc lourd ». La décision de dévaluer (ou non) est ainsi appelée à ne pas dépendre exclusivement de la trajectoire prévisible du processus d'ajustement.

L'application rapide de ces mesures a pour effet de réduire l'inflation (en 1959, 1960 et 1961, elle atteint respectivement $5,7 \%, 3,5 \%$ et 3,4\%), le différentiel d'inflation vis-à-vis de l'Allemagne (premier partenaire commercial de la

\footnotetext{
4 Ancien directeur du Budget dans les années 1950, Roger Goetze est alors conseiller économique du général de Gaulle ; Georges Pompidou est son directeur de Cabinet.

5 En 1957 déjà une « dévaluation masquée » du franc est intervenue (c'est l'opération 20 \% : taxes à l'importation et détaxes à l'exportation).

6 Selon Jean-Marcel Jeanneney, au sein du Comité, Jacques Rueff n'était pas chaud partisan de la dévaluation, c'est la Banque de France qui a surtout milité pour cette issue (Entretien avec JeanMarcel Jeanneney, Paris, le 11 octobre 2000).
} 
France) tend à devenir négligeable en 1961. Une compétitivité prix retrouvée provoque dès 1959 un renversement durable - jusqu'en 1964 - du solde commercial et du solde courant. La dévaluation et « ses » mesures d'accompagnement n'entravent pas pour autant l'élan de la croissance française, au contraire, semble-t-il, puisque le rythme de la croissance s'accélère entre 1959 et 1962 . Une situation de quasi plein emploi continue de prévaloir.

Les souvenirs frais de la dévaluation de 1958 constituent une référence majeure de toutes discussions ultérieures. Si la plupart des analystes reconnaissent le succès de la dévaluation de 1958 et sa capacité à être un instrument de croissance, certains, à l'instar de Jean-Marcel Jeanneney (membre du Comité Rueff), pensent «que l'économie française aurait été capable de se rétablir sans cela» (1997, p.106). Les réserves de Jean-Marcel Jeanneney sont déterminées par une hostilité de principe envers l'inflation. Pour lui, la dépréciation de la valeur interne d'une monnaie mine les fondements même de la société ; ses effets redistributifs sont par nature préjudiciables ${ }^{7}$.

A ce stade de l'étude, plusieurs autres questions méritent d'être posées. En tant que conseiller économique, Rueff accepte d'inscrire « son action » dans le cadre d'un système monétaire différent de celui qu'il appelle de ses vœux, pourquoi fait-il ainsi un compromis avec son projet radical de monétarisme métallique ? Voit-il immédiatement en De Gaulle un allié pour promouvoir plus tard une nouvelle organisation des relations économiques internationales? Pourquoi de Gaulle choisit-il, quant à lui, la «voie Rueff », de fait, une forme de soumission à la contrainte externe alors que les analyses d'influence «cambridgienne » de M. Byé sont synonymes d'une certaine capacité de l'économie nationale à s'affranchir structurellement - c'est-à-dire durablement - de cette contrainte : ce projet économique est a priori en accord avec la vision gaullienne de la souveraineté de la France. De Gaulle a-t-il, lui aussi, des vues à plus long terme et est-il dès 1958 (peut-être déjà sous l'influence de Rueff ?) partisan d'un retour à l'étalon-or classique ? A-t-il déjà l'intention de demander la conversion des dollars de la Banque de France en or? Bref, sa pensée métalliste exprimée avec force lors de la fameuse conférence de presse de février $1965^{\circ}$ est-elle déjà en place ?

Sans pour autant clore le débat théorique sur l'efficacité d'une dévaluation, l'expérience de 1958 paraît donner momentanément raison à J. Rueff qui garantissait au général De Gaulle le retour à l'équilibre de la balance des paiements « en très peu de semaines » ${ }^{9}$ sous réserve d'une application intégrale de son plan,

\footnotetext{
7 Entretien avec Jean-Marcel Jeanneney, Paris, 11 octobre 2000.

8 Rappelons les propos très explicites du général de Gaulle : «La loi suprême, la règle d'or c'est bien le cas de le dire - qu'il faut remettre en vigueur et en honneur dans les relations économiques internationales, c'est l'obligation d'équilibrer, d' une zone monétaire à l'autre, par rentrées et sorties effectives de métal précieux, la balance des paiements résultant de leurs échanges » (Extraits de la conférence de presse du général De Gaulle du 4 février 1965, cité par Bourguinat (1999, p. 537).

9 Le général de Gaulle convoque Jacques Rueff et le questionne sur son plan : «Toutes vos recommandations sont excellentes. Mais si je les applique intégralement et qu'il ne se passe rien, aura-t-il valu vraiment la peine de bouleverser le pays? J' ai noté le soir même ma réponse : “Je vous affirme, mon Général, que le plan, s'il est intégralement appliqué rétablira en très peu de semaines
} 
plutôt qu'à Maurice Byé qui se montrait très réservé quant à son efficacité. M. Byé affirmait ainsi, début 1959, "le commerce extérieur national est régi par des élasticités-prix faibles dont la somme, avoisinant l'unité, est propice au jeu des effets “pervers" » (1959, p. 179). Il soulignait surtout alors l'importance prééminente des structures de la croissance interne en matière de relations économiques internationales et partant la nécessité pour l'Etat de promouvoir des spécialisations avantageuses, autant de dispositions qui exigent du temps.

L'année 1962 voit resurgir des tensions inflations : trois facteurs principaux peuvent être mis en avant pour rendre compte du phénomène.

L'existence d'une pression salariale forte dans le secteur privé et - par ricochet - dans le secteur public. Au sein de l'économie française, la hausse des salaires réels est alors supérieure à l'accroissement de la productivité du travail.

La progression de la masse monétaire du fait d'une augmentation effrénée des crédits à l'économie. L'on touche ici à la principale lacune du plan Rueff qui n'a pas abordé le thème du crédit.

Enfin le « choc exogène ${ }^{10}$ du rapatriement de 800000 Français d'Algérie qui entraîne un accroissement de la demande globale sans que l'offre réagisse.

Le plan de stabilisation de 1963 axé sur un retour à la rigueur budgétaire et un double contrôle des prix et du crédit parvient sans difficulté à rétablir les équilibres monétaire et financier.

Heureuse économie française, dont les autorités paraissent avoir un contrôle si étroit et que seule une perturbation exogène d'envergure semble pouvoir éloigner de son sentier de «croissance d'équilibre ». Même la forte récession de l'économie allemande en 1967 (son produit national connaît un recul absolu) ne réduit que très faiblement le rythme de croissance de la production française $(4,7 \%$ contre 5,2\% en 1966). Malgré une tendance ascendante depuis 1964, le chômage demeure faible en valeur absolue et, comme le souligne Jean-Pierre Vesperini (1993), l'inflation est en définitive contenue puisque, en quatre ans (de janvier 1964 à décembre 1967), le différentiel d'inflation vis-à-vis de l'Allemagne n'atteint que 1,8\% (sur la période, les prix à la consommation ont augmenté de 11,6\% en France contre 9,8\% en RFA).

Mais le choc de 1968 va déséquilibrer profondément l'économie française. Les grèves paralysent presque totalement la production pendant cinq semaines et creusent le déséquilibre externe (accroissement des importations, affaissement des exportations, évasion de capitaux du fait de la crise de régime et, partant, chute des réserves de change). Les accords de Grenelle, par qui vient « la sortie de crise », accordent d'importants avantages sociaux : en juin, le SMIG est élevé de 35\%, les allocations et les retraites sont revalorisées... Pour absorber ce choc d'offre et soulager la trésorerie des entreprises, une politique de crédit accommodante est mise

l'équilibre de notre balance des paiements. De cela je suis absolument sûr ; j'accepte que l'opinion que vous aurez de moi à l'avenir dépende entièrement du résultat obtenu " " (Rueff, 1977, p. 233).

${ }^{10}$ Plus que les événements sociaux de mai 1968, le rapatriement des 800000 Français d'Algérie mérite le qualificatif de choc exogène. 
en œuvre au cours de l'été par le gouvernement Couve de Murville. Les tensions inflationnistes sont avivées malgré les mesures gouvernementales d'aides aux entreprises (diminution de la fiscalité, subventions directes) destinées à couper court aux mécanismes d'auto-entretien de la dynamique ascendante des prix. Au total pour 1968, le différentiel d'inflation avec l'Allemagne atteint 3,6\%. La perte de compétitivité des produits français associée à une « relance isolée » dans un contexte d'interdépendance croissante des économies entraîne l'apparition simultanée d'un déficit commercial et d'un déficit courant. Dans le même temps, malgré la mise en place d'un contrôle des changes (du 6 juin au 5 septembre) les anticipations d'une dévaluation du franc et la poursuite de la fuite des capitaux (alimentées par l'excès de liquidités) aggravent la diminution des réserves de change.

Suite aux événements de mai 68 la question de l'opportunité d'un ajustement monétaire capable de rétablir l'équilibre externe se trouve ouvertement posée, elle occupera les esprits pendant plusieurs mois. Après moins de dix ans de stabilité, il faut reparler d'une dévaluation du franc. Malgré la réussite de 1958, le mot effraye toujours autant l'opinion publique : loin d'être considéré comme une thérapeutique, il est au contraire synonyme d'amputation d'une « substance nationale » dont il est bien difficile d'obtenir la définition. En principe moins en proie aux fétichismes monétaires, les économistes doivent jeter un regard plus clinique sur cette question. Qu'en disent-ils au juste?

\section{3) Opportunité d'une dévaluation et ajustement de la balance des paiements : positions du débat théorique parmi les économistes français}

En France, depuis l'entre-deux-guerres, Jacques Rueff rejette l'idée keynésienne de l'existence d'un niveau naturel d'exportation dépendant des structures de l'économie et qu'il serait, par conséquent, extrêmement difficile de modifier arbitrairement à court et moyen termes. Dans l'ouvrage Le lancinant problème de la balance des paiements, il affirme au contraire : "la balance des paiements ne peut être en déficit durable que si les influences monétaires qui tendent à la mettre en équilibre ont été annihilées ou empêchées d'exercer leurs effets » (1965, p. 23). Le problème de l'équilibre de la balance des paiements n'en est pas un, ou plutôt il devient celui de l'efficacité du système monétaire international, celui de sa capacité d'autorégulation. Le régime de Bretton Woods doit être rejeté en raison de son caractère asymétrique : ce sont les Etats-Unis qui sont à l'origine des problèmes de déséquilibre externe ailleurs dans le monde. Le système leur offre la possibilité de financer leurs déficits par simple création monétaire, par l'émission de «faux droits » et cela sans coût en termes d'activité. Jacques Rueff réclame un retour à l'or qui aurait pour effet de lier échanges extérieurs, contreparties de la base monétaire et niveaux des prix. Comme avant 1913, des « règles » seraient au dessus des nations et imposeraient une double rigueur budgétaire et monétaire. Chez Jacques Rueff, l'équilibre externe s'impose de manière pressante et contraint le niveau de l'activité interne. En 1958, il n'a pas hésité à recommander des mesures d'économie budgétaire afin de prévenir le retour des tensions inflationnistes et de garantir la stabilité du nouveau franc. Le Plan avait ainsi déclenché la fureur de la gauche et de membres du gouvernement (Mollet, Boulloche...) qui redoutaient une contraction de l'activité. 
Le projet radical de Rueff est partagé par De Gaulle. Dès 1961, sous l'influence de son conseiller (voir Jeanneney 1994), il a commencé à exiger la conversion en métal des dollars détenus par la Banque de France. Il entend ainsi mettre au jour les défauts du système de Bretton Woods (« déficit sans pleurs » des Etats-Unis, tendance inflationniste par duplication des bases monétaires) et affirmer la souveraineté monétaire de la France.

Toujours dans la veine des partisans du « laisser-faire » un autre courant paraît souhaiter la dévaluation. Au plus fort des discussions sur l'opportunité d'une dévaluation M. Giboin, J.-D. Lafay et P. Salin (1969) expriment le point de vue des monétaristes français. Le pays ne peut connaître l'expansion que si une politique monétaire « accommodante » est mise en œuvre ; au plan externe, une dévaluation ou mieux encore l'adoption d'une régime de parfaite flexibilité des changes doit automatiquement conduire au retour à l'équilibre de la balance des paiements par le simple jeu des mécanismes de prix. L'accroissement de l'offre de monnaie doit être « calé » sur les besoins de la production et progresser régulièrement.

Partisans d'un flottement pur, les auteurs n'envisagent pas les effets déstabilisants de la spéculation sur le marché des changes et la possible existence d'une dynamique endogène des cours (voir les travaux d'Aftalion 1927, Keynes 1936 et Nurkse 1944). Compte tenu de l'expérience de flottement des années 1920, comment ne pas envisager l'existence de mécanismes explosifs d'interaction entre valeur externe et interne de la monnaie ? Ce silence peut être interprété comme une adhésion implicite aux arguments de M. Friedman (1953) sur le caractère stabilisant de l'action des spéculateurs.

Dans la veine de l'Ecole de Cambridge, pour M. Byé, B. Lassudrie-Duchêne ou encore J. Weiller, les déterminants de l'équilibre externe (l'équilibre est plus ici un concept instrumental qu'un objectif de politique économique) sont fondamentalement d'ordre structurel. La définition de l'équilibre externe est empruntée à Ch. Kindleberger «l'équilibre est cet état de la balance des paiements pendant la période considérée qui la rend capable d'affronter une économie ouverte sans sous-emploi permanent de façon permanente » (Lassudrie-Duchêne (1967, p. 7). Une stratégie de rééquilibrage doit s'appuyer sur une politique industrielle active, la recherche de spécialisations avantageuses fondées notamment sur des économies d'échelle externes ${ }^{11}$ afin de mieux en assurer la pérennité ; bref il s'agit d'un travail de longue haleine qui oblige à penser les modalités même du développement. Pas étonnant dès lors que les positions de F. Perroux puissent être rapprochées de ce courant lui qui fustige la «vision chimérique d'un monde régi par les décisions individuelles des balances des paiements équilibrées et rééquilibrées par les prix, des répartitions de trafics selon les coûts comparatifs des produits » ([1969], p.720) et pour qui le commerce international d'un pays est profondément structuré par ses pôles de développements (ensemble d'unités motrices qui exerce des effets d'entraînement dans un secteur et un espace donné).

La critique de l'approche «néoclassique » passe par un examen attentif des élasticités - prix des importations et des exportations. Les auteurs français mettent

${ }^{11}$ Dans le débat, le concept est sous-jacent mais il n'a bien entendu pas encore été « théorisé » en commerce international, il faudra pour cela attendre les travaux de Paul Krugman au tournant des années 1980 . 
en avant l'existence de possibles effets pervers de nature à contrarier l'ajustement par les prix (réactions par l'instauration de barrières douanières notamment). Tenant d'une approche inductive, Jean Weiller [1969] se penche sur l'expérience de flottement du franc dans les années 1920 pour chercher à invalider les analyses monétaristes. Il constate que la dépréciation des cours de change n'a pas été de pair avec l'équilibre de la balance des paiements, bien au contraire.

L'analyse de ceux que nous appelons les post-keynésiens français conduit à être plus sceptique quant à l'opportunité d'une dévaluation alors que cette mesure est en principe pour les néokeynésiens synonyme d'expansion par le canal d'un accroissement de la demande globale si l'on est à un équilibre de sous-emploi.

Mais voyons maintenant quel écho rencontrent ces développements analytiques auprès des décideurs de l'époque, au moment où les événements monétaires se précipitent.

\section{LA PART DES CHOSES : ARGUMENTS ÉCONOMIQUES ET FACTEURS POLITIQUES DANS LA MARCHE VERS LA DEVALUATION}

Nous allons concentrer notre attention sur les débats de politique monétaire des années 1968 et 1969 afin de déterminer dans quelle mesure les arguments économiques et politiques permettent d'expliquer le timing de la dévaluation. Chemin faisant deux hypothèses seront examinées : nous nous demanderons si la crainte de tensions inflationnistes n'a pas joué un rôle important dans la non-dévaluation de novembre 1968 et si l'élection de Pompidou - un Président favorable à la dévaluation - n'a pas, à partir d'avril 1969, ancré les anticipations de changes et précipité la marche vers la dévaluation.

\section{Une aversion inflationniste derrière la non dévaluation de 1968 ?}

En novembre 1968 la mise en lumière de la réalité de la situation économique française entraîne une très forte tension sur le marché des changes, «les mots de "panique", de "débâcle" viennent sous la plume des commentateurs. » (Lacouture, 1985, t. 3, p. 734). Les opérateurs anticipent une dévaluation. La dynamique de marché se nourrit de toutes les rumeurs. La dévaluation serait, dit-on, de $10 \%$. Ce chiffre a été, semble-t-il, avancé par le ministre des Finances François-Xavier Ortoli en petit comité, la rumeur a été reprise dans la presse. Selon la Banque de France (favorable à la réévaluation du mark) les déclarations imprudentes de certains responsables monétaires allemands (dont Hankel chef du département monétaire et du crédit au Ministère allemand de l'économie ${ }^{12}$ ) seraient à l'origine

${ }^{12}$ Selon les responsables de la Banque de France, Hankel «s'était en effet livré à des déclarations fort imprudentes, début novembre, lors de la réunion du comité central du marché financier à Francfort, en présence de banquiers, qui, comme on le sait, gardent rarement pour eux les confidences qu' ils reçoivent dans les réunions de ce genre. Il avait indiqué que les efforts du Gouvernement de Bonn ne pourraient aboutir à une stabilité monétaire satisfaisante qu'avec le soutien des banquiers et que, 
de cette crise de change : en excluant trop ouvertement l'hypothèse d'une réévaluation du mark, les Allemands auraient activé le mouvement de défiance vis-àvis du franc. L'hostilité de la Banque de France envers la dévaluation du franc nous apparaît historiquement comme une manifestation supplémentaire de sa culture de la stabilité monétaire. Toujours au rang des rumeurs, les journaux affirment que l'octroi d'éventuels crédits européens destinés à soutenir le franc serait subordonné à la décision de dévaluer. Seule information digne de foi : le 19 novembre le ministre allemand des Finances annonce que, contrairement aux bruits qui circulent et au souhait (en coulisses) des autorités monétaires françaises, la RFA ne réévaluera pas le mark. L'hypothèse d'une dévaluation devient de plus en plus probable. L'opposition de gauche autour de Mitterrand, Ortoli et Chirac au sein du gouvernement ainsi que les fonctionnaires de la rue de Rivoli y sont favorables. Tous en attendent des effets expansionnistes afin d'éloigner le spectre du chômage : le cadre de référence ou plutôt de rattachement semble être ici la synthèse keynéso-classique (modèle IS/LM ouvert).

La vieille garde gaulliste semble peu à peu se résoudre à cette issue. Lorsque Jean-Marcel Jeanneney rencontre Maurice Couve de Murville tôt dans la matinée du 23 novembre, ce dernier lui déclare : «la décision de dévaluer est prise »(Jeanneney, 1997, p. 256).

Cette journée du samedi 23 est décisive pour l'avenir du franc, les adversaires de la dévaluation jouent leurs dernières cartes et parviennent à différer le cours de l'histoire monétaire française.

Roger Goetze voit le général De Gaulle et lui déclare : «Vous n'avez pas de chance de réussir parce que d'abord le taux de dévaluation - que je ne connais pas mais à ce qu'on dit - me paraît faible, donc insuffisant pour créer une dynamique de succès. D'autre part, il n'y a pas de plan. »(Goetze 1997, p. 365).

A 11 heures Jean-Marcel Jeanneney est reçu par De Gaulle. Pour convaincre le Président de la République il avance d'abord des arguments politiques. Il rappelle au général de Gaulle qu'après avoir déclaré quelques jours plus tôt (le 13 novembre) en Conseil des ministres que la dévaluation était absurde, «il ne pouvait pas, sans perdre toute autorité et prestige, accepter qu'elle ait lieu » (1997, p. 256). En liaison étroite avec son ancien directeur de cabinet R. Barre, maintenant vice-président de la Commission européenne ${ }^{13}$, Jeanneney peut aussi

faute de cet appui, les autorités allemandes risqueraient de se trouver dans la nécessité de réévaluer le deutsche mark, solution qui n'était pas sans inconvénients pour la République Fédérale. Cette prise de position suivait il faut bien le dire beaucoup d'autres affirmations aussi maladroites de diverses personnalités allemandes selon lesquelles le deutsche mark ne serait pas réévalué pour l'instant, et, en tout cas, ne le serait qu' en dernier ressort. C'est assurément à la suite de l' exposé de M. Hankel que l'agitation, déjà assez grande, est devenue très forte. » (Archives de la Banque de France, Procès Verbal du Conseil Général, le 28 novembre 1968.)

${ }^{13}$ Cette volonté de la Commission européenne de soutenir une issue non inflationniste à la crise française de novembre 1968 semble corroborer un certain nombre d'idées avancées lors du colloque «Le rôle des ministères des Finances et des ministères de l'Economie dans la construction européenne (1957-1978) » tenu à Bercy les 26, 27 et 28 mai 1999. La doctrine des milieux européens était alors déjà libérale, ils rejetaient fermement le dirigisme et l'inflation. Pour un haut fonctionnaire français imprégné de préceptes interventionnistes un passage dans les organismes européens constituait " une école de réalisme ». Les progrès de la construction européenne ont d'ailleurs sans doute été un facteur d'avancée des idées libérales dans la haute fonction publique française. 
affirmer que les prêts européens seront accordés à la France même si elle ne dévalue pas. Il souligne, par ailleurs, que R. Barre est lui aussi hostile à la dévaluation. Les deux hommes redoutent les effets inflationnistes d'un décrochage du franc. Pour résoudre le problème de la compétitivité des entreprises françaises, J.-M. Jeanneney esquisse auprès du général De Gaulle les grandes lignes d'une réforme fiscale combinant baisse de la fiscalité sur les salaires et progression de la TVA, il ne s'agit ni plus ni moins que d'une dévaluation déguisée. Le général de Gaulle paraît sensible à l'argumentation ${ }^{14}$.

C'est ensuite au tour d'Alain Prate de se faire l'avocat de la stabilité : effet psychologique désastreux de la mesure, risque d'une entrée dans un cercle vicieux inflationniste...

Ces conseils d' « hommes de $1958 »$ rejoignent sans doute les aspirations profondes du Général de Gaulle en matière monétaire. Pour lui, le prestige de la France, notamment face aux Etats-Unis, va de pair la préservation de la valeur externe du franc. Au cours des années 1960, cette stabilité externe se trouve élevée au rang d'objectif à part entière pour, soi-disant, préserver le crédit du pouvoir. De même, le Président de la République manifeste une profonde aversion vis-àvis de l'inflation, Alain Peyrefitte rapporte à ce propos des prises de position sans ambiguïté : «Il tient à son franc. Il voudrait le voir garder son bon poids. Mais il le sent menacé par l' inflation. Celle-là, il la hait viscéralement. » (1994, p. 531). Plus loin, toujours dans la bouche de De Gaulle, la sémantique est sans ambiguïté : «l'opium de l'inflation » ou encore «le monstre de l'inflation» (1994, p. 537). Contrairement à un mythe tenace qui vaudrait que la période soit marquée par une inflation ouverte voire galopante, les années 1960 se caractérisent en réalité par une croissance relativement modérée du niveau général des prix, ainsi entre 1960 et 1967 le taux de croissance annuel moyen des prix à la consommation ne dépasse pas 3,5\%.

La crainte de tensions inflationnistes nous apparaît comme l'un des facteurs qui a influencé la décision du Président de la République de ne pas dévaluer. Au total, cependant, c'est bien à la combinaison (aux pondérations insondables) d'un ensemble d'arguments de nature à la fois politique et économique qu'il faut attribuer le renversement d'opinion du général de Gaulle.

Le Conseil des ministres extraordinaire, réuni l'après-midi du 23, décide donc de maintenir la parité du franc. Quelques jours plus tard un sondage IFOP montre que $62 \%$ des Français approuvent cette décision : la dimension symbolique du change et la fascination tenace pour la « monnaie forte » sont susceptibles d'expliquer ce résultat.

Cette résistance monétaire s'accompagne d'un retour à la rigueur. Economies budgétaires, encadrement du crédit et rétablissement - dès le 25 novembre - d'un contrôle rigoureux des changes en constituent les lignes de forces. La réforme fiscale avancée par Jean-Marcel Jeanneney est mise en œuvre : le $1^{\text {er }}$ décembre la taxe sur les salaires est supprimée, les taux de la TVA sont ensuite relevés passant d'une grille de 6 à $20 \%$ à une grille de 7 à $25 \%$. Ce transfert de charges fiscales des entrepreneurs vers les ménages va dans le sens d'une restauration de la compétiti-

14 Entretien avec Jean-Marcel Jeanneney, Paris, 11 octobre 2000. 
vité externe des entreprises françaises en même temps qu'il revient de manière furtive sur les accords de Grenelle.

La poursuite de la hausse des prix du fait notamment de nouvelles pratiques en matière d'indexation salariale réduit plus encore la compétitivité des produits français et contribue à creuser le déficit commercial (6,4 milliards de francs en 1969 contre 1,5 en 1968) et le déficit courant (10,4 milliards en 1969, contre 6,8 en 1968). La volonté gaullienne de maintenir la parité du franc est néanmoins jugée crédible par les opérateurs : début 1969, aucune tension comparable à celle de novembre ne se fait jour sur le marché des changes, l'on observe même un mouvement de retour des capitaux évadés (près d'un milliard de francs au cours du premier trimestre).

\section{Election de Pompidou, ancrage des anticipations de change et dévaluation}

Après le départ du général De Gaulle (le 28 avril 1969), J. Chaban-Delmas premier chef de gouvernement du Président Pompidou - procède à une dévaluation de 11,1\% (9 août 1969), le taux est modéré mais ses effets sont renforcés par la réévaluation du mark allemand le 27 octobre de la même année. Une très large majorité de la classe politique salue l'opportunité du moment et la pertinence de la mesure. L'on en attend une restauration rapide de la compétitivité des entreprises françaises et un retour à l'équilibre externe. Cette fois les rares adversaires de la dévaluation n'ont pas pu intervenir. Dans Le Figaro du 13 août, J.-M. Jeanneney ne peut que critiquer sévèrement cette décision. La situation présente est dit-il «inverse de celle de 1958 ». Au sein d'une économie en «surchauffe » (qui utilise pleinement ses capacités productives), l'accroissement de la demande du fait de la dynamisation du commerce extérieur ne peut qu'activer la hausse des prix. Il y a risque d'entrer dans le cercle vicieux des dévaluations à répétition. Et l'ancien ministre de conclure son propos « une volonté affirmée et crédible de ne plus désormais céder aux tentations de politiques d'abandon sera demain plus nécessaire que jamais ». J.-M. Jeanneney est rejoint dans sa critique par les communistes français pour qui «la dévaluation était évitable au prix de mesures énergiques contre la spéculation et pour consacrer des ressources financières au développement de la production, conformément aux besoins nationaux ». Le PC craint que la dévaluation soit « surtout un mauvais coup contre les travailleurs et toutes les petites gens 》(Le Monde, 13 août 1969) du fait du renchérissement du coût de la vie. Il nous semble que cette position communiste peut être rattachée à la fois à l'idée que les structures d'une économie nationale sont importantes pour expliquer sa situation externe et à la vision néo-keynésienne de possibles effets inflationnistes par excès de demande. Le gouvernement est conscient des éventuelles conséquences inflationnistes de la dévaluation et multiplie les déclarations rassurantes, ainsi V. Giscard d'Estaing - ministre de l'économie et des finances déclare-t-il qu' "il ne faut pas croire que la dévaluation doit s'accompagner automatiquement d'une hausse des prix intérieurs »(Le Monde, 12 août 1969).

La crainte de tensions sur les prix anime l'élaboration d'un plan d'accompagnement qui cherche à opérer une substitution des composantes de la demande 
globale : l'optique de l'absorption est ici sous-jacente, la perspective est toujours néo-keynésienne. La hausse de la demande externe doit être compensée par un ralentissement de la consommation mais sans réduction du pouvoir d'achat des ménages afin d'élever le taux d'épargne, de soutenir par là même l'investissement et d'atteindre ainsi une plus forte croissance de la production. Les politiques de crédit à l'investissement ont un caractère ouvertement sélectif c'est-à-dire structurel. Dans la lignée des travaux de J. Mistral [1975], M. Aglietta et C. Baulant insistent sur le fait que la dévaluation de 1969 a été « l'instrument d'une politique de modernisation industrielle pour renforcer l'économie française dont l'ouverture augmentait très vite» (1993, p. 507). Sur la question de l'ajustement de la balance des paiements, l'approche régulationniste paraît rejoindre la vision keynésienne : toujours est-il qu'effectivement le plan de 1969 semble aussi inspirer par une approche en termes de structures. Comment ne pas repenser aux propos de M. Byé en conclusion de son commentaire de l'opération de décembre 1958 : «Il faut souligner le rôle capital que sont appelés à jouer les investissements publics comme stimulant, comme moyen de régulation et d'orientation de l'économie » (1959, p. 198).

Cela étant c'est bien une compétitivité-prix retrouvée qui explique le retour immédiat à l'équilibre externe. Et Jean-Charles Asselain de constater la réussite spectaculaire de l'opération : «Non seulement le déficit commercial de 1968-1969 est très vite résorbé, mais en plus, contrairement à la tendance antérieure le redressement est particulièrement marqué à l'égard des échanges avec la CEE et les autres pays industrialisés. Les exportations, avec un taux de croissance record de 13,3\% par an (en volume) de 1969 à 1973, jouent plus nettement que jamais un rôle moteur dans la croissance française » (1984, pp. 136-137). La dévaluation de 1969 est un succès car si, toujours sous l'effet de la pression salariale, les tensions inflationnistes demeurent fortes, la croissance connaît un dynamisme exceptionnel : sur la période 1969-1972, elle atteint des maxima historiques, le produit intérieur brut progresse en moyenne de 5,4\%. Cette compétitivité est préservée du fait de la montée de l'inflation chez les principaux partenaires commerciaux de la France.

Si l'ensemble des analystes s'accordent à souligner la pertinence de la décision et, au final, la réussite de la dévaluation, peu nombreux sont ceux qui s'interrogent sur les origines « circonstancielles » du décrochage du franc.

Jean-Marcel Jeanneney ${ }^{15}$ invite à voir dans l'élection de Pompidou à la Présidence de la République le facteur déclenchant d'une fuite de capitaux qui rendait la dévaluation de l'été 1969 inévitable. Selon lui, l'élection d'un Président favorable à une dévaluation a constitué un élément d'ancrage des anticipations de change dans le sens d'une baisse du franc.

Même si, comme son Premier Ministre Jacques Chaban-Delmas, Georges Pompidou s'était officiellement prononcé pour le maintien de la parité du franc, en coulisses ses tendances « dévaluationnistes » n'étaient un secret pour personne. Dès la fin 1968, il avait clairement affiché sa préférence pour la dévaluation. Jean-

${ }^{15}$ Entretien avec Jean-Marcel Jeanneney, Paris, le 11 octobre 2000. 
Marcel Jeanneney se souvient qu'après les événements du 23 novembre, lors d'une réception à l'Assemblée Nationale, il déclarait à Jacques Chirac devant une dizaine de personnes « au moins vous, vous avez soutenu la dévaluation! » Les positions de Pompidou étaient bien connues dans les milieux d'affaires dont il restait proche. Après coup, en août 1969, il confirmait que depuis juillet 1968 il était convaincu que la dévaluation était inévitable (Le Monde, 13 août 1969).

L'étude des mouvements de capitaux corrobore cette analyse : le second trimestre de l'année 1969 est marqué par une reprise massive de la fuite des capitaux.

Cet événement peut être analysé dans le cadre des travaux de Rogoff (1985) qui relient le caractère plus ou moins conservateur du responsable monétaire aux anticipations concernant la valeur de la monnaie.

Sur le plan des influences théoriques, nous avons constaté le caractère prégnant de la grille de lecture keynéso-classique derrière les prévisions des conséquences d'une dévaluation du franc en 1969. L'argument de l'inflation par la demande a été au centre de beaucoup de prises de position durant l'été 1969. Ces craintes inflationnistes ont influencé les modalités du plan d'accompagnement de la dévaluation du franc. Ces prises de position postulent la répercussion entière des variations du change sur les prix des importations et des exportations. L'importance des structures économiques est également ressortie du plan révélant, sans surprise, la prédominance des idées keynésiennes en cette fin des années 1960. Les analyses monétaristes doivent à leur caractère « radical » (retour à l'étalon-or classique dans un cas, adoption d'un régime de changes flexibles dans l'autre) de ne pas avoir influencé de manière décisive le débat français sur l'ajustement. Reste à voir maintenant la manière dont les faits rétroagissent sur les développements de la théorie économique au début des années 1970.

\section{III. ÉVOLUTIONS THÉORIQUES DE LA QUESTION DE L'AJUSTEMENT : UNE RÉACTION AU DÉCROCHAGE DU DOLLAR DE 1971}

L'analyse des travaux sur la question de l'ajustement au cours des années 1970 montre la grande vitalité du débat et fait apparaître un effort d'adaptation de la théorie à un environnement monétaire international beaucoup plus instable. Le cas français ne constitue pas un point d'appui empirique central dans ce mouvement de recomposition essentiellement anglo-saxon. Le facteur déclenchant de l'évolution théorique réside, sans conteste, dans le décrochage du dollar en 1971.

La plus grande instabilité de la valeur externe de la monnaie nous semble impulser deux prolongements théoriques importants. D'une part, elle contribue à une meilleure connaissance de la combinaison des effets prix et des effets quantités en arrière plan de la synthèse keynéso-classique, d'autre part elle conduit à l'intégration des variations du change dans le modèle monétaire de la balance des paiements du Fonds monétaire international. 


\section{Vers une analyse plus fine des effets d'une dévaluation, les nouvelles exigences du rééquilibrage}

Au cours de la première moitié des années 1970 les travaux de W. H. Branson (1972), H. B. Junz et R. R. Rhomberg (1973), R. Masera (1973) et S. P. Magee (1973), (1974) éclairent la nature de la combinaison - jusque là assez obscure des effets prix et quantités à la suite d'une dévaluation. Ils analysent la vitesse du processus d'ajustement et les obstacles susceptibles de l'entraver.

Branson étudie les conséquences de la dépréciation du dollar fin 1971 sur le solde commercial américain. Il montre que l'existence d'une élasticité imparfaite (non infinie) de l'offre conduit à un changement de prix dans les échanges internationaux moins que proportionnel à la variation initiale du change.

L'étude empirique large (un échantillon de treize pays industrialisés est considéré sur la période 1953-1969) de Junz et Rhomberg fait apparaître que les dévaluations ont des effets sur le solde commercial mais qu'ils interviennent souvent avec retard (parfois trois ans) d'où l'impression d'inefficacité de la mesure.

Prenant acte du décrochage du dollar, Masera étudie plus en détail le processus d'ajustement. Il s'appuie sur la dévaluation de la livre sterling en novembre 1967 pour montrer que le redressement de la balance commerciale n'est pas immédiat. Il peut être schématisé sous le trait d'une « courbe en $\mathrm{J}$ ». Un décrochage monétaire provoque dans un premier temps (environ une année) une dégradation du solde commercial de par l'effet négatif de la détérioration des termes de l'échange. L'effet-prix l'emporte alors sur l'effet-quantité, pour des raisons comptables. C'est seulement dans un second temps que le regain de compétitivité prix permet de gagner des parts de marché et d'observer une amélioration progressive de ce solde. L'effet quantité prenant dès lors l'ascendant sur l'effet prix.

Magee s'appuie lui sur l'exemple américain (toujours la dévaluation du dollar de 1971) pour chercher à encore mieux cerner les diverses séquences de ce processus d'ajustement. Il identifie lui aussi une première phase de «currencycontract » au cours de laquelle les contrats signés avant le dévaluation-dépréciation régissent les échanges commerciaux internationaux et partant expliquent la dégradation du solde. L'apparition d'un redressement du solde commercial dépend de la phase cruciale dite de 'pass-through'. Il s'agit de voir ici quelle part de la variation du change est effectivement répercutée sur les prix des exportations et des importations autrement dit de tenir compte des comportements de marge. Plus ceux-ci sont importants, moins le redressement sera vif. Il apparaît que les comportements de marge rendent plus exigeantes les conditions d'élasticités critiques.

Ces travaux pionniers qui ont par la suite donné naissance à une abondante littérature, nous paraissent prolonger l'approche néokeynésienne de l'ajustement de la balance des paiements en nuançant davantage encore le caractère automatique de l'ajustement à la suite d'une dévaluation. Après les événements de 1971, on cesse d'assimiler modification du change et modification des prix des exportations et des importations. 


\section{L'évolution du modèle monétaire de la balance des paiements, l'intégration du change}

Avec la fin de la convertibilité-or du dollar l'approche métalliste de la balance des paiements semble perdre du terrain au profit de l'approche monétaire portée notamment par l'équipe du FMI. Le modèle monétaire témoigne alors de sa capacité d'adaptation : lui aussi est influencé par les événements monétaires de la fin des années 1960 et du début des années 1970.

Le modèle créé par le Fonds monétaire international à la fin des années 1950 étudiait les effets sur la formation du revenu et la balance des paiements de deux variables exogènes : les variations autonomes des exportations et la création interne de crédit bancaire, autrement dit deux sources de variations exogènes de l'offre de monnaie. Le caractère synthétique du modèle apparaît dès l'origine avec cette double intégration de facteurs réel et monétaire.

Le modèle se présente de la sorte :

$$
\mathrm{dMO}=\mathrm{k} \cdot \mathrm{dY}
$$

La variation de l'offre de monnaie d'un pays (dMO) est proportionnelle à la variation de son revenu, le facteur k représentant l'inverse de la vitesse de circulation de la monnaie.

$$
\mathrm{M}=\mathrm{m} . \mathrm{Y}
$$

La demande d'importations (M) est fonction du revenu, m représente la propension marginale à importer du pays (l'on trouve ici un autre élément de nature keynésienne).

$$
\mathrm{dMO}=\mathrm{dR}+\mathrm{dD}
$$

La variation de l'offre de monnaie (dMO) est égale à la variation des réserves extérieures de l'économie nationale à la laquelle on ajoute la variation du crédit intérieur du système bancaire $(\mathrm{dD})$.

$$
\mathrm{dR}=\mathrm{X}-\mathrm{M}+\mathrm{K}
$$

La variation des réserves extérieures $(\mathrm{dR})$ est, quant à elle, égale au solde exportations moins importations majorées de l'entrée nette de capitaux du secteur non bancaire $(\mathrm{K})$.

A l'origine le modèle appréhendait deux éléments caractéristiques de l'économie internationale : l'accroissement tendancielle de la demande mondiale et la fixité des cours de change héritée de Bretton Woods. Les turbulences monétaires de la fin des années 1960 puis le développement du flottement poussent l'équipe du FMI à ajouter le change à l'équation d'importations. Pour garantir que le cours de change reste assez concurrentiel pendant la durée d'un accord de confirmation Polak (1997) rappelle que le FMI y inclut le niveau minimum de réserves internationales que le pays bénéficiaire doit conserver pour pouvoir effectuer des tirages spéciaux car on estime que le pays utilisera le cours de change pour respecter ce niveau minimum. L'ancien directeur des Etudes du FMI précise : «La condition- 
nalité type du FMI en est donc venue à prévoir une double obligation monétaire : fixer un plafond à l' expansion des avoirs intérieurs de la banque centrale afin que le solde de la balance des paiements soit acceptable (concept de flux) et un plancher à ses avoirs extérieurs nets (concept de stock) pour parvenir à un niveau satisfaisant de réserves extérieures, et veiller à ce que la banque centrale $n$ 'intervienne pas excessivement pour s'opposer aux pressions du marché visant à déprécier davantage le taux de change » (1997, pp. 17-18).

L'intégration du taux de change dans le modèle constituait, selon Polak, une nécessite non seulement en raison des effets de ses variations sur les flux commerciaux mais aussi en raison des anticipations inflationnistes. Les autorités ont en effet le choix entre deux possibilités ; soit l'adoption d'un taux flottant pour neutraliser l'impact inflationniste d'une offre excédentaire de "monnaie d'origine étrangère », soit la fixité des taux (parités ajustables) pour fournir un ancrage nominal au plan interne au risque de perdre de la compétitivité s'il reste de l'inflation inertielle dans l'économie.

\section{CONCLUSION}

Vis-à-vis des interrogations de départ concernant les liens entre théories et faits monétaires, l'étude met au jour l'existence d'interactions plutôt diffuses.

Ainsi le personnel politique ne nous paraît pas adhérer de manière explicite et entière à un cadre d'analyse. La référence est souvent lointaine, assez édulcorée. Parmi les analyses disponibles du processus d'ajustement de la balance des paiements à la suite d'une dévaluation, la classe politique semble imprégnée des enseignement du cadre de « la synthèse keynéso-classique ». Les responsables retiennent l'idée, assez intuitive, qu'une dévaluation améliore la compétitivité des produits nationaux et peut rétablir le solde courant. Cette mesure n'est efficace que si la demande globale est inférieure à l'offre globale, dans le cas contraire elle accélère l'inflation.

Les théoriciens semblent de leur côté un peu plus prompts à réagir à l'apparition de faits nouveaux. Les bouleversements monétaires du tournant des années 1970 ont entraîné certaines évolutions analytiques : une meilleure connaissance du processus d'ajustement (principalement à travers l'étude de la liaison variation du change / variation des prix de ventes) au sein de la « synthèse » et l'intégration du change dans le modèle monétaire de la balance des paiements. Notons cependant que les dévaluations de la livre sterling et du franc français paraissent avoir été considérées comme des épiphénomènes. C'est le décrochage du dollar en 1971 qui agit comme un révélateur de la plus grande instabilité des cours de change et pousse les économistes (les principaux apports sont l'œuvre d'anglo-saxons) à amender leurs modèles.

Il y a en définitive comme une exigence de masse critique pour que des faits parviennent à impulser une recomposition analytique (il faut qu'ils soient significativement nouveaux). De même, seule l'orthodoxie théorique la plus solidement établie paraît susceptible d'influencer les responsables politiques et de constituer un point de repère dans le guidage de la pratique monétaire. 


\section{BIBLIOGRAPHIE}

Aftalion, Albert, Monnaie, prix et change, Paris, Sirey, 1927.

Aglietta, Michel et Baulant, Camille, « Le franc : de l'instrument de croissance à la recherche de l'ancrage nominal », in Du franc Poincaré à l' Ecu, CHEFF, pp. 503-553, 1993.

Argy, Victor, The Postwar International Money Crisis, London, George Allen \& Unwin Ltd, 1981. Asselain, Jean-Charles, Histoire économique de la France, Paris, Seuil, tome II, 1984.

Asselain, Jean-Charles, «Le siècle des dévaluations. Du franc Poincaré au "franc fort" ", Les Notes Bleues, 2002.

Bevant, Didier, « De l'opération de décembre 1958 au discours sur le crédit (décembre 1961) de Jacques Rueff », in Du Franc Poincaré à l’ Ecu, CHEFF, pp. 471-497, 1993.

Bourguinat, Henri, Finance internationale, Paris, Puf, 1999.

Branson, William. H, « The Trade Effects of the 1971 Currency Realigments », Brookings papers on Economic Activity, $\mathrm{n}^{\circ}$ 1, pp. 15-58, 1972.

Bussière, Eric, « La Banque de France et la réforme du système monétaire international : entre impératifs nationaux et solidarités des Banques centrales européennes (1963-1968) », Histoire Economie et Sociétés, $\mathrm{n}^{\circ}$ 4, pp. 797-814, 1999.

Byé, Maurice, «L'opération des 27-28 décembre 1958 », Revue Economique, n 2, pp. 161-200, 1959.

Byé, Maurice, Relations économiques internationales, Paris, Dalloz, 1965.

De Grauwe, Paul, La monnaie internationale, Paris, De Boeck, 1999.

Friedman, Milton, Essays on Positive Economics. The Case for Flexible Exchange Rates, Washington, 1953.

Giboin, Maurice, Lafay, Jean-Dominique et Salin, Pascal, Le franc contre l'expansion, Paris, Cujas, 1969.

Goetze, Roger, Entretiens avec Roger Goetze haut fonctionnaire des Finances, Paris, CHEFF, 1997, entretiens conduits par Nathalie Carré de Malberg.

Jeanneney, Jean-Marcel, « De Bretton Woods à la Jamaïque : contestations françaises », Economie Internationale, $\mathrm{n}^{\circ}$ 59, pp. 55-70, 1994.

Jeanneney, Jean-Marcel, Une mémoire républicaine. Entretiens avec Jean Lacouture, Paris, Seuil, 1997.

Johnson, Harry et Frenkel, Jacob (eds), The Monetary Approch to the Balance of Payments, London, George Allen \& Unwin Ltd, 1976.

Junz, Helen B. et Rhomberg, Rudolf R., « Price Competitiveness in Export Trade among Industrial Countries », American Economic Review, Papers and Proceedings, vol. 63, n 2, pp. 412-418, 1973.

Keynes, John-Maynard, Economic Articles and Correspondance, The Collected Writing of John-Meynard Keynes, Cambridge, vol. XI, 1983.

Keynes, John-Maynard, Théorie générale de l'emploi de l'intérêt et de la monnaie, Paris, Payot, traduction française, 1971.

Kindleberger, Charles, International Economics, Georgetown, Richard D. Irwin, 1973.

Kindleberger, Charles, Histoire financière de l'Europe occidentale, Paris, Economica, 1990.

Lacouture, Jean, De Gaulle, Paris, Seuil, tomes II et III, 1985.

Lassudrie-Duchène, Bernard, « Déséquilibre extérieur et contrôle de l'activité intérieure », Revue de science financière, $\mathrm{n}^{\circ} 3$, pp. 357-374, 1959.

Lassudrie-Duchène, Bernard, Economie internationale, Cours Université de Bordeaux, 1967.

Magee, Stephen P., «Currency Contracts, Pass-through, and Devaluation », Brookings Papers on Economic Activity, ${ }^{\circ}$ 1, pp. 303-323, 1973.

Magee, Stephen P, «U.S. Import Prices in the Currency-Contract Period », Brookings Papers on Economic Activity, $\mathrm{n}^{\circ}$ 1, pp. 117-164, 1974. 
Masera, Rainer, «The J-Curve : UK Experience after the 1967 Devaluation », Metroeconomica, vol. 26, pp. 40-62, 1974.

Mistral, Jacques, «Vingt ans de redéploiement du commerce extérieur », Economie et Statistique, $\mathrm{n}^{\circ} 71$, octobre 1975 .

Nurkse, Ragnar, International currency experience, League of Nations, Princeton University Press, 1944.

Patat, Jean-Pierre et Lutfalla, Michel, Histoire monétaire de la France au XXe siècle, Paris, Economica, 1986.

Perroux, François, L'économie du XXe siècle, Grenoble, Pug, 1991.

Peyrefitte, Alain, C'était De Gaulle, Paris, Fayard, tome 1, 1994.

Polak, Jacques J., «Le modèle monétaire du FMI. Un outil toujours précieux », Finances et Developpement, décembre, pp. 16-19, 1997.

Prate, Alain, La France et sa monnaie. Essai sur les relations entre la Banque de France et les gouvernements, Paris, Julliard, 1987.

Rogoff, Kenneth, « The Optimal Degree of Commitment to an Intermediat Monetary Target », Quaterly Journal of Economics, pp. 1169-1189, 1985.

Rueff, Jacques, Le lancinant problème de la balance des paiements, Paris, Payot, 1965.

Rueff, Jacques, De l'aube au crépuscule, Paris, Plon, 1977.

Saint-Marc, Michelle, Histoire monétaire de la France 1800-1980, Paris, Puf, 1983.

The Monetary Approch to the Balance of Payments, IMF, Washington, 1977.

Vesperini, Jean-Pierre, L'économie française sous la Ve République, Paris, Economica, 1993.

Weiller, Jean, «Flexibilité ou fluctuation des changes? », Cahiers de l' ISMEA, n 3, pp. 657-677, mars 1969. 